PROCEEDINGS OF THE

AMERICAN MATHEMATICAL SOCIETY

Volume 138, Number 2, February 2010, Pages 605-614

S 0002-9939(09)10062-X

Article electronically published on September 25, 2009

\title{
A NOTE ON CR MAPPINGS OF POSITIVE CODIMENSION
}

\author{
JEAN-CHARLES SUNYÉ
}

(Communicated by Franc Forstneric)

\begin{abstract}
We prove the following Artin type approximation theorem for smooth CR mappings: given $M \subset \mathbb{C}^{N}$ a connected real-analytic $\mathrm{CR}$ submanifold that is minimal at some point, $M^{\prime} \subset \mathbb{C}^{N^{\prime}}$ a real-analytic subset, and $H: M \rightarrow M^{\prime}$ a $\mathcal{C}^{\infty}$-smooth $\mathrm{CR}$ mapping, there exists a dense open subset $\mathcal{O} \subset M$ such that for any $q \in \mathcal{O}$ and any positive integer $k$ there exists a germ at $q$ of a real-analytic CR mapping $H^{k}:(M, q) \rightarrow M^{\prime}$ whose $k$-jet at $q$ agrees with that of $H$ up to order $k$.
\end{abstract}

\section{INTRODUCTION}

Given germs of real-analytic submanifolds $M$ and $M^{\prime}$ embedded in complex spaces, a fundamental question is to decide whether the formal equivalence of $M$ and $M^{\prime}$ implies their biholomorphic equivalence. While this need not be in general the case in view of a well known example due to Moser-Webster [12] (see also [7, 8]), recent results due to Baouendi, Mir, Rothschild and Zaitsev [5, 3] provide a partial positive answer when the submanifolds are furthermore assumed to be CR. In [5, 3, the positive solution is obtained by approximating in the Krull topology a given formal holomorphic equivalence by a convergent one, following the spirit of Artin's approximation theorem 11. In this paper, we prove the following Artin type approximation theorem for arbitrary smooth CR mappings of any positive codimension.

Theorem 1.1. Let $M \subset \mathbb{C}^{N}$ be a connected real-analytic $C R$ submanifold that is minimal at some point, $M^{\prime} \subset \mathbb{C}^{N^{\prime}}$ be a real-analytic subset, and $H: M \rightarrow M^{\prime}$ be a $\mathcal{C}^{\infty}$-smooth $C R$ mapping. Then there exists a dense open subset $\mathcal{O} \subset M$ such that for any $q \in \mathcal{O}$ and any positive integer $k$ there exists a germ at $q$ of a real-analytic $C R$ mapping $H^{k}:(M, q) \rightarrow M^{\prime}$ whose $k$-jet at $q$ agrees with that of $H$ up to order $k$.

Here minimality is meant in the sense of Tumanov (see Section 2 for the precise definition). To the author's knowledge, Theorem 1.1 is the first result of its kind for mappings of positive codimension between arbitrary real-analytic submanifolds. When the target is a real-algebraic set instead of a real-analytic set, then Theorem 1.1 follows from the work of Meylan, Mir and Zaitsev [10. Observe that

Received by the editors March 16, 2009.

2000 Mathematics Subject Classification. Primary 32V10, 32H02, 32V20.

Key words and phrases. CR mapping, Artin approximation theorem.

The author was partially supported by the Amadeus program of the "Partenariat Hubert Curien".

(c)2009 American Mathematical Society Reverts to public domain 28 years from publication 
Theorem 1.1 is also new even in the case $N=N^{\prime}$ since there is no rank assumption on the mapping under consideration (compare with [5, 3, 13]). On the other hand, we do not know whether one may choose in Theorem 1.1 the dense open subset $\mathcal{O} \subset M$ to be a Zariski open subset independent of the mapping $H$. Note that when $M^{\prime}$ is real-algebraic, such a choice is possible and follows from the main result of [10. For more details related to Artin type approximation in CR geometry, we refer the reader to the survey paper [1].

In this paper we shall give a rather elementary and self-contained proof of Theorem 1.1. For this, we will use several main steps of [6] for which we will provide simplified proofs of the results needed for this paper.

We will organize the paper as follows. Section 2 contains some basic definitions and technical lemmas used in Section 3 . In Section 3. we give some elementary properties of a complex-analytic set invariantly attached to a graph of a smooth CR-mapping. The last section is devoted to the proof of Theorem 1.1.

\section{Preliminaries}

In this section we first recall some basic definitions and prove a lemma used in Section 3. For basic background on CR analysis, we refer the reader to [2. Let $M \subset \mathbb{C}^{N}$ be a real-analytic generic submanifold of codimension $d$. Let us recall that $M$ is said to be minimal at $p \in M$ if there is no germ of a real submanifold $S \subset M$ through $p$ such that the complex tangent space of $M$ at $q$ is tangent to $S$ at every $q \in S$ and $\operatorname{dim}_{\mathbb{R}} S<\operatorname{dim}_{\mathbb{R}} M$ (see [2]).

Following [6], for a $\mathcal{C}^{\infty}$-smooth CR mapping $f: M \rightarrow \mathbb{C}^{N^{\prime}}$ and for $p \in M$, we denote $\mathcal{T}_{p}(f)$ as the germ of the smallest complex analytic set in $\mathbb{C}^{N+N^{\prime}}$ containing the germ of the graph of $f$ at $(p, f(p))$. The integer $\operatorname{dim} \mathcal{T}_{p}(f)-N$ will be called the degree of partial analyticity of $f$ at $p$ and denoted by $\operatorname{deg}_{p} f$. We may observe that, if $M$ is minimal at $p$, the degree of partial analyticity of $f$ at $p$ is non-negative (see Remark 3.2).

We will need the following well known reflection principle (see e.g. [4, 9]).

Proposition 2.1 (Reflection principle). Let $M$ be a real-analytic generic submanifold in $\mathbb{C}^{N}$ minimal at some point $p \in M$. Assume that $u: M \rightarrow \mathbb{C}$ is a continuous $C R$ function near $p$ in $M$ and that $\Theta:\left(\mathbb{C}^{2 N+1},(p, \bar{p}, \overline{u(p)})\right) \rightarrow \mathbb{C}$ is the germ at $(p, \bar{p}, \overline{u(p)})$ of a holomorphic function such that the function $F(z)=\Theta(z, \bar{z}, \overline{u(z)})$ is $C R$ near $p$ in $M$. Then there exists a unique holomorphic extension of $F$ near $p$ in $\mathbb{C}^{N}$.

In what follows, we say that a $\mathcal{C}^{\infty}$-smooth mapping $h: \Omega \rightarrow \mathbb{C}^{l}$, with $\Omega$ being a real manifold, is not identically zero near a point $p_{0}$ if the germ of $h$ at $p_{0}$ is non-zero, i.e. if we find points $p$ as close as we want to $p_{0}$ such that $h(p) \neq 0$.

We will also use the notion of wedge. For $p \in M$, we consider an open neighborhood $U$ of $p$ in $\mathbb{C}^{N}$ and a local defining real-analytic function $\rho: U \rightarrow \mathbb{R}^{d}$ of $M$ near $p$. If $\Gamma$ is an open convex cone in $\mathbb{R}^{d}$ with vertex at the origin, an open set $\mathcal{W}$ of the form $\{z \in U, \rho(z, \bar{z}) \in \Gamma\}$ is called a wedge of edge $M$ in the direction $\Gamma$ centered at $p$.

The following result is a lemma from [6] for which we provide a more elementary proof. 
Lemma 2.2. Let $M \subset \mathbb{C}^{N}$ be a real-analytic generic submanifold, minimal at $p \in$ $M$, let $F:(M, p) \rightarrow \mathbb{C}^{s}$ and $u:(M, p) \rightarrow \mathbb{C}^{t}$ be two germs of $\mathcal{C}^{\infty}$-smooth $C R$ mappings and let $\psi:\left(\mathbb{C}^{2 N+t+s},(p, \bar{p}, \overline{u(p)}, F(p))\right) \rightarrow \mathbb{C}$ be a germ at $(p, \bar{p}, \overline{u(p)}, F(p))$ of a holomorphic function. Assume that $\psi(z, \bar{z}, \overline{u(z)}, F(z)) \equiv 0$ for $z \in M$ near $p$ and that the function $(z, w) \mapsto \psi(z, \bar{z}, \overline{u(z)}, w)$ is not identically zero near $(p, F(p))$ in $M \times \mathbb{C}^{s}$. Then there exists $q \in M$ as close as we want to $p$ such that $\operatorname{deg}_{q} F<s$.

Proof. This result will be proved by induction on the integer $s$. First, we consider the case where $s=1$.

Let $A$ be the set of points $p_{1}$ near $p$ in $M$ such that the holomorphic function $\mathbb{C} \ni w \mapsto \psi\left(p_{1}, \overline{p_{1}}, \overline{u\left(p_{1}\right)}, w\right)$ is not identically zero near $F\left(p_{1}\right)$ in $\mathbb{C}$. We may find $p_{1} \in A$ as close as we want to $p$. Indeed, since $\psi(z, \bar{z}, \overline{u(z)}, w)$ is not identically zero near $(p, F(p))$ in $M \times \mathbb{C}$, there exists $\left(p_{1}, p_{1}^{\prime}\right)$ as close as we want to $(p, F(p))$ such that $\psi\left(p_{1}, \overline{p_{1}}, \overline{u\left(p_{1}\right)}, p_{1}^{\prime}\right) \neq 0$. So, the holomorphy of $\mathbb{C} \ni w \mapsto \psi\left(p_{1}, \overline{p_{1}}, \overline{u\left(p_{1}\right)}, w\right)$ implies it cannot be identically zero near $F\left(p_{1}\right)$ in $\mathbb{C}$. Moreover, for $z \in A$ fixed, since the holomorphic function $\mathbb{C} \ni w \mapsto \psi(z, \bar{z}, \overline{u(z)}, w)$ doesn't vanish identically near $F(z)$ in $\mathbb{C}$, there exists a unique positive integer $k_{z}$ such that $\frac{\partial^{k z} \psi}{\partial w^{k z}}(z, \bar{z}, \overline{u(z)}, F(z)) \neq 0$ and, for any integer $k<k_{z}, \frac{\partial^{k} \psi}{\partial w^{k}}(z, \bar{z}, \overline{u(z)}, F(z))=0$. Now we fix a sufficiently small open neighborhood $V$ of $p$ in $M$ and consider the integer

$$
K=\min \left\{k_{z}, z \in A \cap V\right\} .
$$

We may pick $p_{1} \in A \cap V$ such that $k_{p_{1}}=K$, and we have

$$
\begin{aligned}
\frac{\partial^{K-1} \psi}{\partial w^{K-1}}\left(p_{1}, \overline{p_{1}}, \overline{u\left(p_{1}\right)}, F\left(p_{1}\right)\right) & =0, \\
\frac{\partial^{K} \psi}{\partial w^{K}}\left(p_{1}, \overline{p_{1}}, \overline{u\left(p_{1}\right)}, F\left(p_{1}\right)\right) & \neq 0 .
\end{aligned}
$$

Since $\psi$ is holomorphic near $(p, \bar{p}, \overline{u(p)}, F(p))$ in $\mathbb{C}^{2 N+t+1}$ by the implicit function theorem, there exists a germ at $\left(p_{1}, \overline{p_{1}}, \overline{u\left(p_{1}\right)}\right)$ of a holomorphic function

$$
\Theta:\left(\mathbb{C}^{2 N+t},\left(p_{1}, \overline{p_{1}}, \overline{u\left(p_{1}\right)}\right)\right) \rightarrow \mathbb{C}
$$

such that the zeros of $\frac{\partial^{K-1} \psi}{\partial w^{K-1}}$ near $\left(p_{1}, \overline{p_{1}}, \overline{u\left(p_{1}\right)}, F\left(p_{1}\right)\right)$ in $\mathbb{C}^{2 N+t+1}$ are given by the equation $w=\Theta(z, \zeta, \nu)$. On the other hand, we may observe that the function $\frac{\partial^{K-1} \psi}{\partial w^{K-1}}(z, \bar{z}, \overline{u(z)}, F(z))$ is identically zero near $p_{1}$ in $M$. Suppose, in order to reach a contradiction, that it is false. In this case, we may find $p_{2}$ as close as we want to $p_{1}$ in $M$ such that $\frac{\partial^{K-1} \psi}{\partial w^{K-1}}\left(p_{2}, \overline{p_{2}}, \overline{u\left(p_{2}\right)}, F\left(p_{2}\right)\right) \neq 0$, so there is a point $p_{2} \in A \cap V$ with $k_{p_{2}}<K$. This is a contradiction in view of the definition of $K$. So $\frac{\partial^{K-1} \psi}{\partial w^{K-1}}(z, \bar{z}, \overline{u(z)}, F(z)) \equiv 0$ for $z \in M$ near $p_{1}$, and, from the remark on the zeros of $\frac{\partial^{K-1} \psi}{\partial w^{K-1}}$, we obtain that $F(z)=\Theta(z, \bar{z}, \overline{u(z)})$ near $p_{1}$ in $M$. But if $p_{1}$ is close enough to $p$, we may assume that $M$ is minimal at $p_{1}$ (since $M$ is real-analytic and minimal at $p$ ), and consequently we may apply Proposition 2.1 to obtain the 
existence of a holomorphic extension $\widetilde{F}$ of $F$ near $p_{1}$ in $\mathbb{C}^{N}$. Thus the graph of $F$ is contained, near $p_{1}$, in the graph of $\widetilde{F}$, which is a complex analytic set of dimension $N$. Consequently, the dimension of $\mathcal{T}_{p_{1}}(F)$ is less than or equal to $N$. So we proved that, for an arbitrary small neighborhood $V$ of $p$ in $M$, there exists $p_{1} \in V$ such that $\operatorname{deg}_{p_{1}} F \leq 0$. This finishes the proof of the lemma for $s=1$.

Now, we assume that the lemma holds for $s-1$, and for any $t \in \mathbb{N}$, any germs of CR mappings $F:(M, p) \rightarrow \mathbb{C}^{s-1}, u:(M, p) \rightarrow \mathbb{C}^{t}$ and any germ at $(p, \bar{p}, \overline{u(p)}, F(p))$ of a holomorphic function $\psi:\left(\mathbb{C}^{2 N+t+s-1},(p, \bar{p}, \overline{u(p)}, F(p))\right) \rightarrow$ $\mathbb{C}$. Our aim is to prove the same result for $s$. We write $w=\left(w^{\prime}, w_{s}\right) \in \mathbb{C}^{s-1} \times \mathbb{C}$ and $F=\left(F^{\prime}, F_{s}\right) \in \mathbb{C}^{s-1} \times \mathbb{C}$.

First, we consider the case where $\psi\left(z, \bar{z}, \overline{u(z)}, F^{\prime}(z), w_{s}\right) \equiv 0$ for $\left(z, w_{s}\right) \in$ $M \times \mathbb{C}$ near $\left(p, F_{s}(p)\right)$. Taking the Taylor series of $\psi$ in $w_{s}$ at $F_{s}(p)$,

$$
\psi\left(z, \zeta, \nu, w^{\prime}, w_{s}\right)=\sum_{k \in \mathbb{N}} \psi_{k}\left(z, \zeta, \nu, w^{\prime}\right)\left(w_{s}-F_{s}(p)\right)^{k},
$$

we obtain that, for any $k \in \mathbb{N}, \psi_{k}\left(z, \bar{z}, \overline{u(z)}, F^{\prime}(z)\right) \equiv 0$ for $z \in M$ near $p$ and that there exists $k_{0}$ such that $\psi_{k_{0}}\left(z, \bar{z}, \overline{u(z)}, w^{\prime}\right)$ doesn't vanish identically near $\left(p, F^{\prime}(p)\right)$ in $M \times \mathbb{C}^{s-1}$. So, by the induction hypothesis, there exists $q \in M$ as close as we want to $p$ such that $\operatorname{deg}_{q} F^{\prime}<s-1$, which implies $\operatorname{deg}_{q} F<s$. This completes the proof for this case.

To finish the proof, we have to consider the case where $\psi\left(z, \bar{z}, \overline{u(z)}, F^{\prime}(z), w_{s}\right)$ doesn't vanish identically near $\left(p, F_{s}(p)\right)$ in $M \times \mathbb{C}$. By the same method as in the case $s=1$, we show that, for point $p_{1}$ as close as we want to $p$ where $M$ is minimal, there exists a germ at $\left(p_{1}, \overline{p_{1}}, \overline{u\left(p_{1}\right)}, F^{\prime}\left(p_{1}\right)\right)$ of a holomorphic function $\Theta:\left(\mathbb{C}^{2 N+t+s-1},\left(p_{1}, \overline{p_{1}}, \overline{u\left(p_{1}\right)}, F^{\prime}\left(p_{1}\right)\right)\right) \rightarrow \mathbb{C}$ such that

$$
F_{s}(z)=\Theta\left(z, \bar{z}, \overline{u(z)}, F^{\prime}(z)\right)
$$

near $p_{1}$ in $M$. Let $\Theta\left(z, \zeta, \nu, w^{\prime}\right)=\sum_{\alpha \in \mathbb{N}^{s-1}} \Theta_{\alpha}(z, \zeta, \nu)\left(w^{\prime}-F^{\prime}\left(p_{1}\right)\right)^{\alpha}$ be the Taylor series of $\Theta$ in $w^{\prime}$ at $F^{\prime}\left(p_{1}\right)$.

If every $\Theta_{\alpha}(z, \bar{z}, \overline{u(z)})$ is CR near $p_{1}$ in $M$, then by Proposition 2.1 (recall that $M$ is minimal at $\left.p_{1}\right)$ the function $M \times \mathbb{C}^{s-1} \ni\left(z, w^{\prime}\right) \mapsto \Theta\left(z, \bar{z}, \overline{u(z)}, w^{\prime}\right) \in \mathbb{C}$ can be holomorphically extended near $\left(p_{1}, F^{\prime}\left(p_{1}\right)\right)$ in $\mathbb{C}^{N+s-1}$. We denote the extension by $\widetilde{\Theta}$. The graph of $F$ is contained in the graph of $\widetilde{\Theta}$, which is a complex submanifold of $\mathbb{C}^{N+s}$ of dimension $N+s-1$. This is equivalent to saying that the degree of partial analyticity of $F$ at $p_{1}$ is smaller than $s-1$.

If there is a multi-index $\alpha \in \mathbb{N}^{s-1}$ such that the mapping $\Theta_{\alpha}(z, \bar{z}, \overline{u(z)})$ is not $\mathrm{CR}$, then there exists a vector field $\bar{L}=\sum_{j=1}^{N} a_{j}(z, \bar{z}) \frac{\partial}{\partial \overline{z_{j}}}$ near $p$ in $\mathbb{C}^{N}$, where $a_{1}, \ldots, a_{N}$ are real-analytic functions near $p$, such that $\left.\bar{L}\right|_{M}$ is a CR vector field and

$$
\bar{L}\left(\Theta\left(z, \bar{z}, \overline{u(z)}, w^{\prime}\right)\right) \not \equiv 0
$$


near $\left(p_{1}, F^{\prime}\left(p_{1}\right)\right)$ in $M \times \mathbb{C}^{s-1}$. Using the chain rule, we may observe that there exists a holomorphic function $\Psi_{1}$ near $\left(p_{1}, \overline{p_{1}},\left.\bar{L}(\overline{u(z)})\right|_{z=p_{1}}, F^{\prime}\left(p_{1}\right)\right)$ in $\mathbb{C}^{2 N+t+s-1}$ such that

$$
\bar{L}\left(\Theta\left(z, \bar{z}, \overline{u(z)}, w^{\prime}\right)\right)=\Psi_{1}\left(z, \bar{z}, \bar{L}(\overline{u(z)}), w^{\prime}\right)
$$

near $\left(p_{1}, F^{\prime}\left(p_{1}\right)\right)$ in $M \times \mathbb{C}^{s-1}$. Since $M$ is minimal at $p$, Tumanov's extension theorem (see [2]) implies that there exists a holomorphic extension $\tilde{u}$ of $u$ in a wedge $\mathcal{W}$ of edge $M$ centered at $p$. We may assume that the mapping $\tilde{u}$ is $\mathcal{C}^{\infty}$ smooth on $\mathcal{W}$ up to the edge $M$. Moreover, for any $z \in \mathcal{W} \cup(M \cap V)$, where $V$ is a sufficiently small neighborhood of $p$ in $\mathbb{C}^{N}$,

$$
\bar{L}(\overline{\tilde{u}(z)})=\sum_{j=1}^{N} a_{j}(z, \bar{z}) \overline{\frac{\partial \tilde{u}}{\partial z_{j}}(z)} .
$$

Now, for any $j \in\{1, \ldots, N\}, \frac{\partial \tilde{u}}{\partial z_{j}}$ is holomorphic in $\mathcal{W}$ and $\mathcal{C}^{\infty}$ up to the edge $M$. Thus, the mapping $U$ whose components are the restrictions to $M$ near $p$ of the derivatives of $\tilde{u}$ is a CR mapping near $p$ in $M$. Consequently, from the identity (2.5), and since $\bar{L}(\overline{u(z)})=\bar{L}(\overline{\tilde{u}(z)})$, for $z \in M$ close enough to $p$, we may find a germ at $\left(p_{1}, \overline{p_{1}}, \overline{U\left(p_{1}\right)}, F^{\prime}\left(p_{1}\right)\right)$ of a holomorphic function

$$
\Psi:\left(\mathbb{C}^{2 N+t+N t+s-1},\left(p_{1}, \overline{p_{1}}, \overline{U\left(p_{1}\right)}, F^{\prime}\left(p_{1}\right)\right)\right) \rightarrow \mathbb{C}
$$

such that

$$
\bar{L}\left(\Theta\left(z, \bar{z}, \overline{u(z)}, w^{\prime}\right)\right)=\Psi\left(z, \bar{z}, \overline{U(z)}, F^{\prime}(z)\right)
$$

near $\left(p_{1}, F^{\prime}\left(p_{1}\right)\right)$ in $M \times \mathbb{C}^{s-1}$. Moreover, we know that $\bar{L}\left(\Theta\left(z, \bar{z}, \overline{u(z)}, F^{\prime}(z)\right) \equiv\right.$ $\bar{L}\left(F_{s}(z)\right) \equiv 0$ near $p_{1}$ in $M$, since $F$ is CR; i.e. $\Psi\left(z, \bar{z}, \overline{U(z)}, F^{\prime}(z)\right) \equiv 0$ near $p_{1}$ in $M$. So, since we saw that $\Psi\left(z, \bar{z}, \overline{U(z)}, w^{\prime}\right) \not \equiv 0$ near $\left(p_{1}, F^{\prime}\left(p_{1}\right)\right)$ in $M \times \mathbb{C}^{s-1}$, the induction assumption implies that the degree of partial analyticity of $F^{\prime}$ is strictly smaller than $s-1$ for points in $M$ as close as we want to $p_{1}$ and therefore as close to $p$. This finishes the proof of Lemma 2.2

As in [6], one gets from Lemma 2.2 the following result.

Lemma 2.3. Let $M \subset \mathbb{C}^{N}$ be a real-analytic generic submanifold, minimal at $p \in M$, let $F:(M, p) \rightarrow \mathbb{C}^{s}$ be a germ of a $C R$ mapping and let

$$
\psi:\left(\mathbb{C}^{2 N+2 s},(p, \bar{p}, \overline{F(p)}, F(p))\right) \rightarrow \mathbb{C}
$$

be a germ at $(p, \bar{p}, \overline{F(p)}, F(p))$ of a holomorphic function. Assume that

$$
\psi(z, \bar{z}, \overline{F(z)}, F(z)) \equiv 0
$$

for $z \in M$ near $p$ and that the function $(z, v, w) \mapsto \psi(z, \bar{z}, v, w)$ is not identically zero near $(p, \overline{F(p)}, F(p))$ in $M \times \mathbb{C}^{2 s}$. Then, there exists $q \in M$ as close as we want to $p$ such that $\operatorname{deg}_{q} F<s$. 
Proof. First, we assume that $\psi(z, \bar{z}, \overline{F(z)}, w)$ does not vanish identically near $(p, F(p))$ in $M \times \mathbb{C}^{s}$. Since $\psi(z, \bar{z}, \overline{F(z)}, F(z)) \equiv 0$ for $z \in M$ near $p$, we may apply Lemma 2.2 and deduce that the degree of partial analyticity of $F$ at $q$ is strictly smaller than $s$ for $q$ arbitrarily close to $p$.

Now we treat the case where $\psi(z, \bar{z}, \overline{F(z)}, w) \equiv 0$ for $(z, w) \in M \times \mathbb{C}^{s}$ near $(p, F(p))$. For this we consider the Taylor series of $\psi$ in $w$ at $F(p)$ :

$$
\psi(z, \zeta, v, w)=\sum_{\alpha \in \mathbb{N}^{s}} \psi_{\alpha}(z, \zeta, v)(w-F(p))^{\alpha} .
$$

The assumption implies that, for any $\alpha \in \mathbb{N}^{s}, \psi_{\alpha}(z, \bar{z}, \overline{F(z)}) \equiv 0$ near $p$ in $M$. However, by assumption, there is a multi-index $\alpha_{0}$ such that $\psi_{\alpha_{0}}(z, \bar{z}, v) \not \equiv 0$ near $(p, \overline{F(p)})$ in $M \times \mathbb{C}^{s}$, and Lemma 2.2 gives the desired result.

\section{Properties of $\mathcal{T}_{p}(H)$}

In this section, we fix a real-analytic generic submanifold $M \subset \mathbb{C}^{N}$ and a $\mathcal{C}^{\infty}$ smooth CR mapping $H: M \rightarrow M^{\prime}$ on $M$ with values in a real-analytic set $M^{\prime} \subset$ $\mathbb{C}^{N^{\prime}}$. We shall give some properties of the degree of partial analyticity of $H$ and of the complex analytic set $\mathcal{T}_{p}(H)$ for $p \in M$. All the results of this section can be found in [6], but since the proofs we shall give are rather elementary compared to [6, we include them in this paper for completeness.

The following lemma is a direct consequence of the boundary uniqueness theorem.

Lemma 3.1. Let $M, M^{\prime}$ and $H$ be as above. If $H$ admits a holomorphic extension $\widetilde{H}$ on a wedge of edge $M$ centered at $p$, then the graph of $\widetilde{H}$ near $(p, H(p))$ is contained in $\mathcal{T}_{p}(H)$.

Remark 3.2. If $M$ is minimal at $p \in M$, Tumanov's extension theorem implies that, in the above setting, there is a unique extension $\widetilde{H}$ of $H$ holomorphic on a wedge of edge $M$ centered at $p$. Thus $\mathcal{T}_{p}(H)$ contains the graph of $\widetilde{H}$ near $(p, H(p))$. This implies that the dimension of $\mathcal{T}_{p}(H)$ at $p$ is greater than $N$, i.e. that the degree of partial analyticity of $H$ at $p$ is non-negative.

The two following lemmas describe the regular points of the complex analytic set $\mathcal{T}_{p}(H)$.

Lemma 3.3. In the above setting, assume that the degree of partial analyticity of $H$ is constant near some point $p$ in $M$. Then there exists an open neighborhood $U_{p}^{1}$ of $p$ in $M$ such that the set $\Sigma_{p}^{1} \subset M \cap U_{p}^{1}$ of points $q \in U_{p}^{1}$ for which $\mathcal{T}_{p}(H)$ is not regular at $(q, H(q))$ is a closed set with empty interior.

Proof. We may find an open neighborhood $U_{p}^{1}$ of $p$ in $M$ on which $\mathcal{T}_{p}(H)$ is a complex analytic set and on which the degree of partial analyticity of $H$ is constant equal to $s$. Let $\Sigma_{p}^{1} \subset M \cap U_{p}^{1}$ be the set of points $q \in U_{p}^{1}$ for which $\mathcal{T}_{p}(H)$ is not regular at $(q, H(q))$. In view of the classical definition of regular points of a complex analytic set, $\Sigma_{p}^{1}$ is a closed subset of $M \cap U_{p}^{1}$. To prove that its interior is empty, assume by contradiction that we may find an open subset $V$ of $M$ contained in $\Sigma_{p}^{1}$. Thus, for any $q \in V$, the graph of $H$ near $(q, H(q))$ is contained in the set of 
the singular points of $\mathcal{T}_{p}(H)$, which is a complex analytic set of dimension strictly smaller than $N+s$. So, the dimension of $\mathcal{T}_{q}(H)$ is also strictly smaller than $N+s$ for any $q \in V$. This is impossible, since the degree of partial analyticity of $H$ is constant equal to $s$ on $V$.

Lemma 3.4. In the above setting, assume that $M$ is minimal at $p \in M$, that the degree of partial analyticity of $H$ is constant equal to $s$ near $p$ in $M$, and write $t=N^{\prime}-s$. Then there are an open neighborhood $U_{p}^{2} \subset U_{p}^{1}$ of $p$ in $M$ (with $U_{p}^{1}$ given by Lemma 3.3) and a closed set with empty interior $\Sigma_{p}^{2} \subset M \cap U_{p}^{2}$ such that, for any $q \in U_{p}^{2} \backslash \Sigma_{p}^{2}$, there are holomorphic coordinates $\left(u^{\prime}, v^{\prime}\right) \in \mathbb{C}^{s} \times \mathbb{C}^{t}$ near $H(q)$ for which $H=(F, G) \in \mathbb{C}^{s} \times \mathbb{C}^{t}$, and a germ at $(q, F(q))$ of a holomorphic mapping $T_{q}:\left(\mathbb{C}^{N+s},(q, F(q))\right) \rightarrow \mathbb{C}^{t}$ such that $\mathcal{T}_{p}(H)$ is given near $(q, H(q))$ by the equation $v^{\prime}=T_{q}\left(z, u^{\prime}\right)$. In particular $\mathcal{T}_{p}(H)$ is regular at $(q, H(q))$.

Proof. Since $M$ is minimal at $p$ and $H$ is CR on $M$, Tumanov's extension theorem implies that there exists a holomorphic extension $\widetilde{H}$ of $H$ in a wedge $\mathcal{W}$ of edge $M$ centered at $p$ which is $\mathcal{C}^{\infty}$-smooth up to the edge. By Lemma 3.1 the graph of $\widetilde{H}$ is contained in $\mathcal{T}_{p}(H)$ near $(p, H(p))$. It means that we may choose an open neighborhood $\Delta$ of $p$ in $\mathbb{C}^{N}$ such that $(z, \widetilde{H}(z)) \in \mathcal{T}_{p}(H)$ for any $z \in \Delta \cap \mathcal{W}$.

On the other hand, if $U_{p}^{1}$ is the open neighborhood of $p$ given by Lemma 3.3 . we define $U_{p}^{2}=U_{p}^{1} \cap \Delta$ and $\Sigma_{p}^{2}=\Sigma_{p}^{1} \cap U_{p}^{2}$. Now, for a fixed point $q \in U_{p}^{2} \backslash \Sigma_{p}^{2}$, $\mathcal{T}_{p}(H)$ is regular at $(q, H(q))$. Moreover, since the degree of partial analyticity of $H$ is constant equal to $s$ on $U_{p}^{1}$, the dimension of $\mathcal{T}_{p}(H)$ at $(q, H(q))$ is $N+s$. So, there exist an open neighborhood $\mathcal{U} \subset U_{p}^{2}$ of $q$ in $\mathbb{C}^{N}$, an open neighborhood $\mathcal{V}$ of $H(q)$ in $\mathbb{C}^{N^{\prime}}$ and a holomorphic mapping $f: \mathcal{U} \times \mathcal{V} \rightarrow \mathbb{C}^{t}$ of rank $t$ at $(q, H(q))$ such that

$$
\mathcal{T}_{p}(H)=\left\{\left(z, z^{\prime}\right) \in \mathcal{U} \times \mathcal{V}, f\left(z, z^{\prime}\right)=0\right\} .
$$

Thus, for $z \in \mathcal{U}^{\prime}=\mathcal{U} \cap \mathcal{W} \cap \widetilde{H}^{-1}(\mathcal{V})$, we have

$$
f(z, \widetilde{H}(z))=0 .
$$

So $\frac{\partial f}{\partial z}(z, \widetilde{H}(z))+\frac{\partial f}{\partial z^{\prime}}(z, \widetilde{H}(z)) \cdot \frac{\partial \widetilde{H}}{\partial z}(z) \equiv 0$ in $\mathcal{U}^{\prime}$. Since the mapping $\widetilde{H}$ is $\mathcal{C}^{\infty}$-smooth up to the edge $M$, we have the following identity:

$$
\frac{\partial f}{\partial z}(z, \widetilde{H}(z))+\frac{\partial f}{\partial z^{\prime}}(z, \widetilde{H}(z)) \cdot \frac{\partial \widetilde{H}}{\partial z}(z) \equiv 0
$$

on $M \cap \mathcal{U}^{\prime}$. But $f$ is of $\operatorname{rank} t$ at $(q, H(q))$ and the identity (3.3) shows that the columns of $\frac{\partial f}{\partial z}(q, H(q))$ are a linear combination of the columns of $\frac{\partial f}{\partial z^{\prime}}(q, H(q))$. Consequently the rank of $\frac{\partial f}{\partial z^{\prime}}(q, H(q))$ is $t$. So, by the implicit function theorem there exist holomorphic coordinates $\left(u^{\prime}, v^{\prime}\right) \in \mathbb{C}^{s} \times \mathbb{C}^{t}$ for which $H=(F, G) \in$ $\mathbb{C}^{s} \times \mathbb{C}^{t}$ and a holomorphic mapping $T_{q}$ near $(q, F(q))$ in $\mathbb{C}^{N+s}$ such that the zeros of $f$ are given by points of the form $\left(z, u^{\prime}, T_{q}\left(z, u^{\prime}\right)\right) \in \mathbb{C}^{N} \times \mathbb{C}^{s} \times \mathbb{C}^{t}$.

For the next result, we will denote $\pi: \mathbb{C}^{N} \times \mathbb{C}^{N^{\prime}} \rightarrow \mathbb{C}^{N}$ and $\pi^{\prime}: \mathbb{C}^{N} \times \mathbb{C}^{N^{\prime}} \rightarrow \mathbb{C}^{N^{\prime}}$ as the canonical projections.

Lemma 3.5. In the above setting, assume that $M$ is minimal at $p \in M$ and that the degree of partial analyticity of $H$ is constant equal to $s$ near $p$ in $M$. Then there are an open neighborhood $U_{p}^{3} \subset U_{p}^{2}$ of $p$ in $M$ (with $U_{p}^{2}$ given by Lemma 3.4) and 
a closed set with empty interior $\Sigma_{p}^{3} \subset M \cap U_{p}^{3}$ such that, for any $q \in U_{p}^{3} \backslash \Sigma_{p}^{3}$, there is a neighborhood $\Omega_{q}$ of $(q, H(q))$ in $\mathbb{C}^{N+N^{\prime}}$ satisfying

$$
\pi^{\prime}\left(\left.\mathcal{T}_{p}(H)\right|_{M \times \mathbb{C}^{N^{\prime}}} \cap \Omega_{q}\right) \subset M^{\prime} .
$$

Proof. Let $\rho^{\prime}: \mathbb{C}^{N^{\prime}} \rightarrow \mathbb{R}^{d^{\prime}}$ be a local defining real-analytic function of $M^{\prime}$ near $H(p)$. Since $H(M) \subset M^{\prime}$, we have the mapping identity

$$
\rho^{\prime}(H(z), \overline{H(z)}) \equiv 0
$$

for $z \in M$ near $p$.

We consider $U_{p}^{2}$ and $\Sigma_{p}^{2}$ respectively as the open neighborhood of $p$ in $M$ and the closed set with empty interior given by Lemma 3.4. Let $U_{p}^{3} \subset U_{p}^{2}$ be a sufficiently small connected neighborhood of $p$ in $M$. Since $M$ is real-analytic and minimal at $p$, the set $\Sigma_{1}$ of points in $U_{p}^{3}$ where $M$ is not minimal is a closed set with empty interior. Thus, $\Sigma_{p}^{3}=\left(\Sigma_{p}^{2} \cap U_{p}^{3}\right) \cup \Sigma_{1}$ is a closed set with empty interior. Moreover, for any $q \in U_{p}^{3} \backslash \Sigma_{p}^{3}, M$ is minimal at $q$, and we may find holomorphic coordinates $\left(u^{\prime}, v^{\prime}\right) \in \mathbb{C}^{s} \times \mathbb{C}^{t}$ near $H(q)$ for which $H=(F, G) \in \mathbb{C}^{s} \times \mathbb{C}^{t}$, and a germ at $(q, F(q))$ of a holomorphic mapping $T_{q}:\left(\mathbb{C}^{N+s},(q, F(q))\right) \rightarrow \mathbb{C}^{t}$ such that $\mathcal{T}_{p}(H)$ is given near $(q, H(q))$ by the equation $v^{\prime}=T_{q}\left(z, u^{\prime}\right)$. If $U_{p}^{3}$ is chosen small enough we may assume that the graph of $H$ is contained near $q \in U_{p}^{3} \backslash \Sigma_{p}^{3}$ in $\mathcal{T}_{p}(H)$; i.e. we have the mapping identity

$$
G(z)=T_{q}(z, F(z))
$$

for $z \in M$ near $q$. We may also assume that the mapping defined by

$$
\Psi\left(z, \zeta, \nu^{\prime}, u^{\prime}\right)=\rho^{\prime}\left(u^{\prime}, T_{q}\left(z, u^{\prime}\right), \nu^{\prime}, \overline{T_{q}\left(\bar{\zeta}, \overline{\nu^{\prime}}\right)}\right)
$$

is holomorphic near $(q, \bar{q}, \overline{F(q)}, F(q))$ in $\mathbb{C}^{2 N+2 s}$ for any $q \in U_{p}^{3} \backslash \Sigma_{p}^{3}$.

From (3.5) and (3.4), we obtain

$$
\rho^{\prime}\left(F(z), T_{q}(z, F(z)), \overline{F(z)}, \overline{T_{q}(z, F(z))}\right) \equiv 0
$$

for $z \in M$ near $q$. Thus, by (3.7), we have

$$
\Psi(z, \bar{z}, \overline{F(z)}, F(z)) \equiv 0
$$

for $z \in M$ near $q$. Since the degree of analyticity of $H$ is constant equal to $s$ on $U_{p}^{3}$, from (3.5), we deduce that the degree of partial analyticity of $F$ is constant equal to $s$ on $U_{p}^{3}$. So, the identity (3.8) and Lemma 2.3 (recall that $M$ is minimal at $q$ ) imply that

$$
\Psi\left(z, \bar{z}, \nu^{\prime}, u^{\prime}\right) \equiv 0
$$

for $\left(z, \nu^{\prime}, u^{\prime}\right) \in M \times \mathbb{C}^{2 s}$ near $(q, \overline{F(q)}, F(q))$. This identity is equivalent to

$$
\rho^{\prime}\left(u^{\prime}, T_{q}\left(z, u^{\prime}\right), \nu^{\prime}, \overline{T_{q}\left(z, \overline{\nu^{\prime}}\right)}\right) \equiv 0
$$

for $\left(z, \nu^{\prime}, u^{\prime}\right) \in M \times \mathbb{C}^{2 s}$ near $(q, \overline{F(q)}, F(q))$. So taking $\nu^{\prime}=\overline{u^{\prime}}$ in (3.10), we obtain that $\left(u^{\prime}, T_{q}\left(z, u^{\prime}\right)\right) \in M^{\prime}$ as soon as $z$ is close enough to $q$ in $M$ and $u^{\prime}$ is close enough to $F(q)$ in $\mathbb{C}^{s}$. This finishes the proof of the lemma.

We conclude this section with a lemma which makes use of the upper semicontinuity of the partial analyticity degree; we leave the details to the reader. 
Lemma 3.6. In the above setting, there exists a closed set with empty interior $\Sigma_{2} \subset$ $M$ such that the degree of partial analyticity of $H$ is constant on each connected component of $M \backslash \Sigma_{2}$.

\section{Proof of Theorem 1.1}

In this section, we keep the assumptions and the notation of Section 3 , i.e we consider a real-analytic generic submanifold $M$ in $\mathbb{C}^{N}$ and a $\mathcal{C}^{\infty}$-smooth CR mapping $H: M \rightarrow M^{\prime}$ where $M^{\prime} \subset \mathbb{C}^{N^{\prime}}$ is a real-analytic set. Theorem 1.1 will be a consequence of the following result.

Proposition 4.1. In the above setting, assume that $M$ is minimal at $p \in M$ and that the degree of partial analyticity of $H$ is constant equal to $s$ near $p$ in $M$. Let $U_{p}^{3}$ and $\Sigma_{p}^{3} \subset M \cap U_{p}^{3}$ be, respectively, the open neighborhood of $p$ in $M$ and the closed set with empty interior given by Lemma 3.5. Then for any $q \in U_{p}^{3} \backslash \Sigma_{p}^{3}$ and for any positive integer $k$ there exists a germ at $q$ of a real-analytic $C R$ mapping $H^{k}:(M, q) \rightarrow M^{\prime}$ whose $k$-jet at $q$ agrees with that of $H$ up to order $k$.

Proof. For any $q \in U_{p}^{3} \backslash \Sigma_{p}^{3}$, we may choose holomorphic coordinates $z^{\prime}=\left(u^{\prime}, v^{\prime}\right) \in$ $\mathbb{C}^{s} \times \mathbb{C}^{t}$ and a germ at $(q, F(q))$ of a holomorphic mapping $T_{q}:\left(\mathbb{C}^{N+s},(q, F(q))\right) \rightarrow$ $\mathbb{C}^{t}$ such that $\mathcal{T}_{p}(H)$ is given by the equation $v^{\prime}=T_{q}\left(z, u^{\prime}\right)$ near $(q, H(q))$. We may also find a neighborhood $\Omega_{q}$ of $(q, H(q))$ in $\mathbb{C}^{N+N^{\prime}}$ such that $\pi^{\prime}\left(\left.\mathcal{T}_{p}(H)\right|_{M \times \mathbb{C}^{N^{\prime}}} \cap \Omega_{q}\right)$ $\subset M^{\prime}$.

We fix a point $q \in U_{p}^{3} \backslash \Sigma_{p}^{3}$ and a positive integer $k$. Since the graph of $H$ is contained in $\mathcal{T}_{p}(H)$ near $(q, H(q))$, we have the following mapping identity:

$$
G(z)=T_{q}(z, F(z))
$$

near $q$ in $M$. Let $F^{k}$ be the $k$-th order Taylor polynomial of $F$ at $q$ (that is holomorphic since $H$ is CR; see [2]). We define the holomorphic mapping $G^{k}$ near $q$ in $\mathbb{C}^{N}$ by setting

$$
G^{k}(z)=T_{q}\left(z, F^{k}(z)\right) .
$$

Thus $H^{k}=\left(F^{k}, G^{k}\right)$ is a holomorphic mapping near $q$ in $\mathbb{C}^{N}$ with values in $\mathbb{C}^{N^{\prime}}$. Moreover, by definition of $H^{k}$ and from (4.1), the $k$ first derivatives of $H^{k}$ at $q$ coincide with that of $H$. So to complete the proof of Proposition 4.1, we have to show that $H^{k}$ sends $M$ into $M^{\prime}$ near $q$. From the definition of $H^{k}$ and the local defining equation of $\mathcal{T}_{p}(H)$ near $(q, H(q))$ in $\mathbb{C}^{N+N^{\prime}}$, we obtain that there exists an open neighborhood $\widetilde{\Omega_{q}}$ of $(q, H(q))$ in $\mathbb{C}^{N+N^{\prime}}$ such that

$$
\left.\mathcal{G}_{H^{k}} \cap\left(M \times \mathbb{C}^{N^{\prime}}\right) \cap \widetilde{\Omega_{q}} \subset \mathcal{T}_{p}(H)\right|_{M \times \mathbb{C}^{N^{\prime}}} \cap \Omega_{q},
$$

where $\mathcal{G}_{H^{k}}$ is the graph of the mapping $H^{k}$. Since $\pi^{\prime}\left(\left.\mathcal{T}_{p}(H)\right|_{M \times \mathbb{C}^{N^{\prime}}} \cap \Omega_{q}\right) \subset M^{\prime}$ by Lemma 3.5. the conclusion of the proposition follows.

Now, we are able to prove our main result, Theorem 1.1

Proof of Theorem 1.1. We first note that Theorem 1.1 holds in the case where $N=1$ or $N^{\prime}=1$. We may therefore assume that $N, N^{\prime} \geq 2$.

We first treat the case where $M$ is generic. Since $M$ is real-analytic and connected, there exists a real-analytic subvariety $\Sigma_{1}$ of $M$ such that $M$ is minimal at each point $p \in M \backslash \Sigma_{1}$. Moreover, from Lemma 3.6 there exists a closed set with empty interior $\Sigma_{2} \subset M$ such that the degree of partial analyticity of $H$ is constant 
on each connected components of $M \backslash \Sigma_{2}$. Thus, $\Sigma=\Sigma_{1} \cup \Sigma_{2}$ is a closed subset of $M$ with empty interior. Fix a point $p \in M \backslash \Sigma$; by Proposition 4.1, we may find an open subset $U_{p}^{3}$ of $p$ in $M$ and a closed set with empty interior $\Sigma_{p}^{3} \subset M \cap U_{p}^{3}$ such that, for every $q \in U_{p}^{3} \backslash \Sigma_{p}^{3}$, the conclusion of Theorem 1.1 holds at $q$. Thus, the set $\mathcal{O}=\bigcup_{p \in M \backslash \Sigma}\left(U_{p}^{3} \backslash \Sigma_{p}^{3}\right)$ does the job, and this finishes the proof of Theorem 1.1 for the generic case.

If $M$ is not generic, for any $p \in M \backslash \Sigma_{1}$ (where $\Sigma_{1}$ again denotes the set of nonminimal points of $M$ ) we may assume, thanks to a local holomorphic change near $p$, that $M=\widetilde{M}_{p} \times\{0\} \subset \mathbb{C}_{z_{1}}^{N-r_{1}} \times \mathbb{C}_{z_{2}}^{r_{1}}$, where $r_{1}$ is a non-negative integer and $\widetilde{M}_{p}$ is a connected real-analytic generic submanifold which is minimal (see 2]). From the generic case treated above, there exists a dense open subset $\widetilde{\mathcal{O}}_{p} \subset \widetilde{M}_{p}$ such that, for any non-negative integer $k$ and any $\tilde{q} \in \widetilde{\mathcal{O}}_{p}$, there exists a germ at $\tilde{q}$ of a real-analytic CR mapping $H_{1}^{k}:\left(\widetilde{M}_{p}, q\right) \rightarrow M^{\prime}$ whose $k$-jet at $\tilde{q}$ agrees with that of $\widetilde{M}_{p} \ni z_{1} \mapsto H\left(z_{1}, 0\right)$. Since $\bigcup_{p \in M \backslash \Sigma_{1}}\left(\widetilde{\mathcal{O}}_{p} \times\{0\}\right)$ is a dense open subset of $M$, the proof of Theorem 1.1 is complete.

\section{REFERENCES}

[1] M. Artin, On the solutions of analytic equations. Invent. Math. 5 (1968), 277-291. MR0232018 (38:344)

[2] M. S. Baouendi, P. Ebenfelt, L. P. Rothschild, Real Submanifolds in Complex Space and Their Mappings. Princeton Math. Series 47, Princeton Univ. Press, 1999. MR 1668103 (2000b:32066)

[3] M. S. Baouendi, N. Mir, L. P. Rothschild, Reflection ideals and mappings between generic submanifolds in complex space. J. Geom. Anal. 12 (2002), no. 4, 543-580. MR.1916859 (2003m:32035)

[4] M. S. Baouendi, H. Jacobowitz, F. Trèves, On the analyticity of CR mappings. Ann. of Math. (2) 122 (1985), no. 2, 365-400. MR808223 (87f:32044)

[5] M. S. Baouendi, L. P. Rothschild, D. Zaitsev, Equivalences of real submanifolds in complex space. J. Differential Geom. 59 (2001), no. 2, 301-351. MR.1908985 (2003k:32051)

[6] S. Damour, Feuilletages holomorphes locaux et analyticité partielle d'applications CR $\mathcal{C}^{\infty}$. Manuscripta Math. 109 (2002), no. 2, 203-222. MR.1935029 (2003h:32048)

[7] X. Huang, Local equivalence problems for real submanifolds in complex spaces. Real Methods in Complex and CR Geometry, Lecture Notes in Math. 1848, Springer, Berlin, 2004, 109-163. MR2087582 (2005j:32041)

[8] X. Huang, W. Yin, A Bishop surface with a vanishing Bishop invariant. Invent. Math. 176 (2009), no 3, 461-520. MR2501295

[9] F. Meylan, N. Mir, D. Zaitsev, Analytic regularity of CR-mappings. Math. Res. Lett. 9 (2002), no. 1, 73-93. MR.1892315 (2003d:32041)

[10] F. Meylan, N. Mir, D. Zaitsev, Approximation and convergence of formal CR-mappings. Int. Math. Res. Not. (2003), no. 4, 211-242. MR1935273 (2004b:32058)

[11] F. Meylan, N. Mir, D. Zaitsev, On some rigidity properties of mappings between CRsubmanifolds in complex space. Journées "Équations aux Dérivées Partielles", Exp. No. XII, 20 pp., Univ. Nantes, Nantes, 2003. MR2050598 (2005a:32040)

[12] J. K. Moser, S. M. Webster, Normal forms for real surfaces in $C^{2}$ near complex tangents and hyperbolic surface transformations. Acta Math. 150 (1983), no. 3-4, 255-296. MR709143 (85c:32034)

[13] J. C. Sunyé, On formal maps between generic submanifolds in complex space. J. Geom. Anal. 19 (2009), no. 4, 944-962.

Laboratoire de Mathématiques RaphaËl Salem, Centre National de la Recherche Scientifique, Université de Rouen, Avenue de l’Université, B.P. 12, 76801 Saint Etienne DU Rouvray, France

E-mail address: jean-charles.sunye@etu.univ-rouen.fr 